\title{
Multiparametric dynamic contrast-enhanced ultrasound imaging of prostate cancer
}

\author{
Rogier R. Wildeboer ${ }^{1} \cdot$ Arnoud W. Postema ${ }^{2}$ Libertario Demi ${ }^{1}$. \\ Maarten P. J. Kuenen ${ }^{3}$ Hessel Wijkstra ${ }^{1,2} \cdot$ Massimo Mischi $^{1}$
}

Received: 25 May 2016 /Revised: 28 November 2016 / Accepted: 1 December 2016 /Published online: 21 December 2016

(C) The Author(s) 2016. This article is published with open access at Springerlink.com

\begin{abstract}
Objectives The aim of this study is to improve the accuracy of dynamic contrast-enhanced ultrasound (DCE-US) for prostate cancer (PCa) localization by means of a multiparametric approach. Materials and Methods Thirteen different parameters related to either perfusion or dispersion were extracted pixel-by-pixel from 45 DCE-US recordings in 19 patients referred for radical prostatectomy. Multiparametric maps were retrospectively produced using a Gaussian mixture model algorithm. These were subsequently evaluated on their pixel-wise performance in classifying 43 benign and 42 malignant histopathologically confirmed regions of interest, using a prostate-based leave-one-out procedure. Results The combination of the spatiotemporal correlation $(r)$, mean transit time $(\mu)$, curve skewness $(\kappa)$, and peak time (PT) yielded an accuracy of $81 \% \pm 11 \%$, which was higher than the best performing single parameters: $r(73 \%), \mu(72 \%)$, and washin time $(72 \%)$. The negative predictive value increased to $83 \% \pm 16 \%$ from $70 \%, 69 \%$ and $67 \%$, respectively. Pixel inclusion based on the confidence level boosted these measures to $90 \%$ with half of the pixels excluded, but without disregarding any prostate or region.

Conclusions Our results suggest multiparametric DCE-US analysis might be a useful diagnostic tool for $\mathrm{PCa}$, possibly
\end{abstract}

Rogier R. Wildeboer

r.r.wildeboer@tue.nl

1 Laboratory of Biomedical Diagnostics, Department of Electrical Engineering, Eindhoven University of Technology, PO-Box 513 , $5600 \mathrm{MB}$ Eindhoven, The Netherlands

2 Department of Urology, Academic Medical Center University Hospital, Meibergdreef 9, 1105 AZ Amsterdam, The Netherlands

3 Philips Research, Philips, Eindhoven, The Netherlands supporting future targeting of biopsies or therapy. Application in other types of cancer can also be foreseen.

Key points

- DCE-US can be used to extract both perfusion and dispersion-related parameters.

- Multiparametric DCE-US performs better in detecting PCa than single-parametric DCE-US.

- Multiparametric DCE-US might become a useful tool for PCa localization.

Keywords Prostate cancer · Ultrasound · Contrast agents · Classification $\cdot$ Multiparametric imaging

$\begin{array}{ll}\text { Abbreviations } \\ \text { AT } & \text { Appearance time } \\ \text { CUDI } & \text { Contrast-ultrasound dispersion imaging } \\ \text { DCE-US } & \text { Dynamic contrast-enhanced ultrasound } \\ \text { FWHM } & \text { Full width half mximum } \\ \text { GMM } & \text { Gaussian mixture model } \\ \text { LDRW } & \text { Local density random walk } \\ \text { mpMRI } & \text { Multiparametric magnetic resonance imaging } \\ \text { NPV } & \text { Negative predictive value } \\ \text { PCa } & \text { Prostate cancer } \\ \text { PPV } & \text { Positive predictive value } \\ \text { PSA } & \text { Prostate-specific antigen } \\ \text { PT } & \text { Peak time } \\ \text { ROC } & \text { Receiver operating characteristic } \\ \text { ROI } & \text { Region of interest } \\ \text { TIC } & \text { Time-intensity curve } \\ \text { TRUS } & \text { Transrectal ultrasound } \\ \text { UCA } & \text { Ultrasound contrast agent } \\ \text { WIT } & \text { Wash-in time }\end{array}$




\section{Introduction}

Prostate cancer (PCa) is the most prevalent form of cancer among American men, representing 26\% of the new cases and approximately $10 \%$ of the cancer-related deaths [1]. Therefore, a reliable and minimally invasive diagnostic tool for PCa is of paramount importance. During the last decade of the 20th century, the introduction of prostate-specific antigen (PSA) blood testing led to a dramatic increase of the number of PCa diagnoses as well as a growing number of patients exhibiting low-risk or indolent disease [2]. Overdiagnosis and overtreatment are considered substantial problems due to the limited positive predictive value (PPV) of screening tools such as PSA level assessment and digital rectal examination $[3,4]$. Therefore, the definitive diagnosis of PCa still relies on $\geq 10$ core systematic biopsy [5]. There is a high incidence of biopsy-related complications $[6,7]$ and a considerable fraction of malignancies is identified only in repeat biopsy [8]. This stresses the demand for an imaging modality that is able to localize or rule out prostatic malignancies. Such a technique could eventually serve as a localization tool for targeted biopsy $[9,10]$, or assist in patient selection and treatment planning for organ-sparing focal therapy [11].

Currently, multiparametric magnetic resonance imaging (mpMRI) seems the most promising imaging method for PCa localization [12]. A recent meta-analysis reported an appreciable average sensitivity of $74 \%$ and specificity of $88 \%$, with negative predictive values (NPVs) that ranged from $65 \%$ to $94 \%$ [13]. In view of the advantages of transrectal ultrasound (TRUS) over MRI in terms of costs, time, resolution, and practicality at bedside, this paper proposes a multiparametric approach of TRUS.

Unfortunately, B-mode TRUS is not considered sufficiently accurate for stand-alone tumour detection $[14,15]$. Since clinically relevant prostatic malignancies are characterized by angiogenesis and neovascularization [16, 17], increased perfusion has been proposed as a marker for PCa. Moreover, it was observed that the microvascular density correlates with cancer aggressiveness [18]. However, Doppler imaging was not found sufficiently accurate to capture these vascular changes due to its limited sensitivity for small flows [19, 20]. The use of intravenously injected ultrasound contrast agents (UCAs) in the TRUS procedure, that is, dynamic contrast-enhanced ultrasound (DCE-US) imaging, also allows the visualization of the vascular fraction and perfusion. Contrast-specific imaging modes are even able to image microcirculation at a capillary scale [21]. Again, despite the reported improvements in tumour detection rate [22, 23], targeted biopsies based on visual interpretation of contrastenhanced US alone are not considered viable to replace systematic biopsy $[9,24]$. This might be explained by the inconsistent, ambiguous effect of angiogenesis on blood flow [25]. Whereas diminished vasomotor control and formation of shunts cause an elevation in perfusion, the high tortuosity and permeability, with rising interstitial pressure, lead to the opposite effect [16, 17].

Tissue characterization by DCE-US thus requires a more detailed assessment of the UCA kinetics in the prostate. UCAs are composed of encapsulated micron-sized gas bubbles that remain a few minutes in the vasculature [26-28], and their behaviour can be assessed by looking at the time-intensity curve (TIC), which is the evolution of echo intensity over time in a certain area. For example, malignant areas in DCE-US recordings are found to be marked by rapid and enhanced inflow compared to similar benign regions in the prostate $[29,30]$. Several parameters have been extracted from the TICs in order to mark relevant alterations in perfusion; these are, e.g., the wash-in rate [31, 32], time to peak (PT) [33, 34], time to appearance (AT) [33, 34], peak intensity (PI) [32-35], and the area under the curve $[33,36]$. In addition to these perfusion-related parameters, angiogenic microvasculature changes can also be detected by assessing UCA dispersion [37]. Contrast US dispersion imaging (CUDI) was recently developed to analyse the dispersion kinetics of a microbubble contrast bolus in a DCE-US recording. The dispersive behaviour has been assessed with curve fitting [37], or similarity analysis [38, 39]. In these methods, the TIC of each pixel in the imaging plane is either fitted by a convective diffusion model or compared to its neighbouring TICs, respectively.

Though different in nature, the parameters acquired with these methods were shown to have appreciable levels of sensitivity and specificity for PCa detection. Hence, we hypothesize that a combination of complementary parameters related to perfusion and dispersion allows us to localize prostatic carcinoma with an even higher level of accuracy. PCa is a multifocal and heterogeneous disease whose appearance depends on cancer type, grade and topography [24]. A multiparametric approach reduces the risk of missing tumours that are invisible to one of the parameters and may be able to discriminate prostatic diseases that mimic malignant characteristics, like prostatitis [40].

Since the performance of a multiparametric approach is not dependent on a single threshold, it cannot be evaluated using conventional receiver operating characteristic (ROC) analysis [41, 42]. Instead, a multiparametric approach requires a classification algorithm to combine the parameters into a single parametric map. Many algorithms have been used in biomedicine for classification; in particular, Gaussian mixture models (GMMs), support vector machines and artificial neural networks have been extensively employed [43-45]. GMMs have been chosen for our multiparametric evaluation as these are fast, purely based on data (no need for additional physical modelling) and facilitate the definition of classification confidence. Moreover, GMMs were reported to perform better than neural networks in mammographic tumour identification [45]. 
In this paper, we take into account thirteen perfusion- and dispersion-related parameters as well as the echo intensity on the TRUS image. Retrospectively, the most useful parameters are selected and combined using histopathologically determined regions of interest (ROIs) in order to improve the accuracy of DCE-US for the localization of PCa.

\section{Materials and methods}

\section{Data acquisition}

Nineteen PCa patients that were scheduled for radical prostatectomy underwent a transrectal DCE-US scan prior to surgery. The procedures were approved by the local ethics committee and carried out at the Academic Medical Center (Amsterdam, The Netherlands). All patients signed an informed consent. Patients below the age of 18 or with contraindications for the administration of contrast agents as defined by the European Medicines Agency were excluded. Patients with a tumour of Gleason score $\geq 3+3$ and a size suitable for our analysis (see section "Histopathological Analysis") were selected for this study. The patient and tumour characteristics are summarized in Table 1.

For the procedure, $2.4 \mathrm{~mL}$ of a SonoVue ${ }^{\circledR}$ UCA microbubble suspension (Bracco, Milan, Italy) was intravenously administered. This suspension consists of encapsulated sulphur hexafluoride bubbles with an average diameter of $2.5 \mu \mathrm{m}$ [46]. Two-minute recordings were subsequently performed with an iU22 US scanner (Philips Healthcare, Bothell, WA, USA), generally using a $3-\mathrm{MHz}$ to $10-\mathrm{MHz}$-ranged endocavity US probe (C10-3v). For one patient, an endocavity probe with a range from $4 \mathrm{MHz}$ to $8 \mathrm{MHz}(\mathrm{C} 8-4 \mathrm{v})$ was used

Table 1 List of patient and tumour characteristics

\begin{tabular}{lccc}
\hline Characteristic & Mean & Median & Range \\
\hline Age (yrs) & 62.7 & 64 & $52-73$ \\
PSA level (ng/mL) & 8.7 & 7.3 & $2.9-31.9$ \\
Prostate volume $(\mathrm{mL})$ & 35.8 & 30 & $20-83.5$ \\
& Number & & \\
Clinical stage & & & \\
T1 & 0 & & \\
T2 & 11 & & \\
T3 & 8 & & \\
Gleason score & & & \\
$3+3$ & 6 & \\
$3+4$ & 7 & \\
$4+3$ & 4 & \\
$3+5$ & 1 & \\
$4+5$ & 1 & \\
\hline
\end{tabular}

due to availability issues. The measurements were carried out in contrast-specific mode based on a power modulation pulse scheme at a frequency of $3.5 \mathrm{MHz}$ and with a mechanical index of 0.06 to minimize bubble disruption.

\section{Histopathological analysis}

After prostatectomy, the prostates were fixed with a formalin solution, deprived of the seminal vesicles, sectioned in slices of $\sim 4 \mathrm{~mm}$ and examined by the pathologist as described by Montironi et al. [47]. The tumours were subsequently delineated by the pathologist. Prior to the parametric analysis, $\sim 0.5 \mathrm{~cm}^{2}$-sized ROIs were manually drawn on the B-mode US scans to identify areas containing histologically confirmed malignancy. Histological images were matched to the US scans based on the position of the imaging plane in a transversal sweep video performed before the contrast recordings. ROIs were only drawn in areas where the histopathologic information persisted in the two adjacent slices. The prostatic boundary was used to aid the localization. In the same way, we positioned similarly sized ROIs in areas that were not depicted as malignant. This resulted in a dataset containing about 174,000 pixels extracted from 85 ROIs in 45 DCE-US recordings in 19 patients, marked as either benign (43 ROIs) or malignant (42 ROIs). In the end, we were able to include one to four DCE-US imaging planes per patient.

\section{Data processing and parameter extraction}

To assess the dispersive UCA behaviour, the DCE-US recordings were analysed by TIC fitting as well as by similarity analysis. The parametric maps were produced using a custom-made CUDI program running in Matlab ${ }^{\circledR}$ (2015b, Mathworks, Natick, MA, USA) [48]. Following the method described in [37], the data were pre-processed and the extracted TICs were fitted by a modified local density random walk (LDRW) model. This allowed us to estimate the area under the curve $(\alpha)$, the mean transit time $(\mu)$, the skewness parameter $(\kappa)$, and the ratio between the diffusive and convective time $(\lambda=\mu \kappa)$. In addition, we looked at the variance (var) which is the second moment of the curve [49], and the fitting interval (int) between the PT and the truncation time where UCA recirculation occurs [37].

For the similarity analysis, the data were pre-processed as described in [39]. The spectral coherence $(\rho)$ [38] and the spatiotemporal correlation coefficient $(r)$ [39] were then calculated. For these parameters, the TIC of a single pixel is compared to those in a ring-shaped kernel of 1.0 to $2.5 \mathrm{~mm}$ in radius, as this size allows us to visualize similarity on the scale of early angiogenesis [38].

Based on the processing in [37], we also extracted the PI, the AT (where the TIC reaches 5\% of the PI) and the PT (the time where the intensity is the highest). In addition, the wash- 
in time (WIT, the time period between AT and the point where the TIC reaches $95 \%$ of the PI), and the full width half maximum (FWHM) were considered as parameters of interest. A full list of the investigated parameters is reported in Table 2 .

\section{Classification procedure}

Gaussian mixture modelling is a widely known approach in cluster analysis [50, 51] and data classification [52, 53]. GMMs describe a set of observations (i.e. pixels) in (multi)parametric space by a mixture of normal distributions. Using two predetermined training subsets of benign and malignant observations, the class-specific probability distributions can be computed. Subsequently, each pixel in the test set is classified according to these distributions. We define a measure for the confidence of classification by comparing the probabilities, $p$, of the observation being benign or malignant. This confidence level, $P$, conveniently ranging from 0 to 1 , is described by $P=\frac{2 p_{A}}{p_{A}+p_{B}}-1$, where $A$ denotes the class with highest probability.

The GMM classification algorithm was implemented in Matlab ${ }^{\circledR}$ using the statistical analysis toolbox. The parameters were normalized to the 90th percentile to ensure equal weighting, and training was performed using an iterative expectation-maximization algorithm [54]. Since the GMM algorithm is very fast, it was feasible to evaluate all possible combinations of one to four distinct parameters. We did not take into account more than four parameters to avoid overfitting.

Table 2 Full list of the parameters considered for multiparametric analysis, with symbols and units

\begin{tabular}{lll}
\hline Symbol & Parameter name & Unit \\
\hline $\begin{array}{l}\text { B-mode ultrasound } \\
\text { Greylevel }\end{array}$ & Echo intensity & a.u. \\
$\begin{array}{l}\text { Contrast-enhanced ultrasound } \\
\text { WIT }\end{array}$ & Wash-in time & $\mathrm{s}$ \\
AT & Appearance time & $\mathrm{s}$ \\
PT & Peak time & $\mathrm{s}$ \\
PI & Peak intensity & a.u. \\
FWHM & Full width half maximum & $\mathrm{s}$ \\
Fitting analysis & & \\
$\kappa$ & Skewness parameter & $\mathrm{s}$ \\
$\mu$ & Mean transit time & $\mathrm{s}$ \\
$\lambda$ & Convective-diffusion ratio & - \\
$\alpha$ & Area under the curve & a.u. \\
var & Variance & a.u. \\
int & Interval time & $\mathrm{s}$ \\
Similarity analysis & & \\
$\rho$ & Spectral coherence & - \\
$r$ & Correlation coefficient & - \\
\hline
\end{tabular}

To evaluate the performance of the classifier, the procedure was tested on each of the prostates whilst using the observations in other prostates as the training set. The outcomes of this leave-one-out analysis were averaged over all prostates. We quantified the classification performance by computing the accuracy, sensitivity, specificity, PPV and NPV [41, 42]. Whereas sensitivity and specificity indicate the percentage of correctly classified malignant and benign pixels, respectively, PPV and NPV reflect the percentage of pixels classified respectively as malignant and benign that were correct. In addition, accuracy represents the overall correct classification percentage. Since the NPV is of paramount importance in order to avoid missing clinically relevant $\mathrm{PCa}$ lesions, we optimized the classifier based on this measure as well as on the accuracy.

\section{Pixel exclusion}

The TIC measurement quality usually differs from pixel to pixel. To ensure the quality of the classification, it is important to identify pixels that are likely to be misclassified. As stated, the classification algorithm indicates the confidence of the classification by the level $P$. Also, we consider the coefficient of determination, $R^{2}$, describing how well the TIC can be fitted by the modified LDRW model, and the absolute probability of an observation belonging to its class. Large healthy vessels are more likely to show early arrival of the bolus, which complicates the use of perfusion parameters to mark malignancy. Therefore, we also evaluated the classifier's performance after excluding the pixels with the lowest PT for each plane.

\section{Results}

Based on accuracy, the combination of $r, \mu, \kappa$, and PTyielded the best accuracy (mean \pm standard deviation $=81 \pm 11 \%$ ). The highest NPV was found for the parameters var, $\mu, r$, and int $(87 \pm 15 \%)$, but with two of the other performance measures being inferior compared to first set. The high NPV and sensitivity can be explained by a low number of false negatives. We found the parameter distributions best described by a single Gaussian function per variable. The outcomes were compared to the performance of individual parameters. The best-performing parameters of all three analyses - $\mu$ for curve fitting, $r$ for similarity analysis and WIT for conventional perfusion analysis - were evaluated by a ROC-based threshold optimization as well as GMM classification in one-dimensional parametric space. As shown in Table 3, the multiparametric classification has a higher performance than the ROC-analysed single parameters, irrespective of the measure used. Since not all individual parameters are well described by a single Gaussian distribution, a non-tailored GMM approach for the single parameters yielded less stable results in terms of the balance between sensitivity and specificity. 
Table 3 Performance of the classification methods using specified parameters

\begin{tabular}{|c|c|c|c|c|c|c|c|}
\hline \multicolumn{3}{|c|}{ ROC Analysis } & \multicolumn{3}{|c|}{ Single GMM } & \multicolumn{2}{|c|}{$\begin{array}{l}\text { Multiparametric } \\
\text { GMM }\end{array}$} \\
\hline WIT & $\mu$ & $r$ & WIT & $\mu$ & $r$ & $\begin{array}{l}r, \mu, \\
\kappa, \mathrm{PT}\end{array}$ & $\begin{array}{l}\text { var, } \mu, \\
r, \text { int }\end{array}$ \\
\hline 72 & 72 & 73 & 73 & 71 & 67 & 81 & 72 \\
\hline 75 & 74 & 71 & 88 & 90 & 65 & 79 & 90 \\
\hline 68 & 70 & 75 & 51 & 47 & 71 & 80 & 50 \\
\hline 76 & 75 & 76 & 70 & 63 & 70 & 85 & 65 \\
\hline 67 & 69 & 70 & 84 & 85 & 72 & 83 & 87 \\
\hline
\end{tabular}

The used abbreviations are listed in the Abbreviations List and Table 2

The best performing perfusion-related parameter as well as best parameters of curve fitting and similarity analysis were evaluated using ROC analysis and single-parameter GMM. Multiparametric results are shown of the parameter sets with the highest accuracy $(r, \mu, \kappa, \mathrm{PT}$ ) and NPV (var, $\mu, r$, int)

Even though accuracy and NPV are considered the most important performance measures, a reliable technique requires the other measures to be sufficiently high as well.

In the "Materials and methods" section, we mentioned feature-based exclusion of pixels to decrease the number of misclassifications in the multiparametric map. Figure 1 shows the changes in accuracy after pixel exclusion based on classification confidence $P$, absolute probability, $R^{2}$, and PT. It reveals that $P$ correctly reflects the confidence and that it is the most suitable measure to identify pixels with a high risk of misclassification. The results of the multiparametric classification after pixel exclusion based on this measure are depicted in Figures $2 \mathrm{a}$ and $\mathrm{b}$ for a parameter set containing $r, \mu, \kappa$, and $P T$ and a set containing var, $\mu, r$, and int, respectively. The exclusion of pixels is equally distributed over benign and

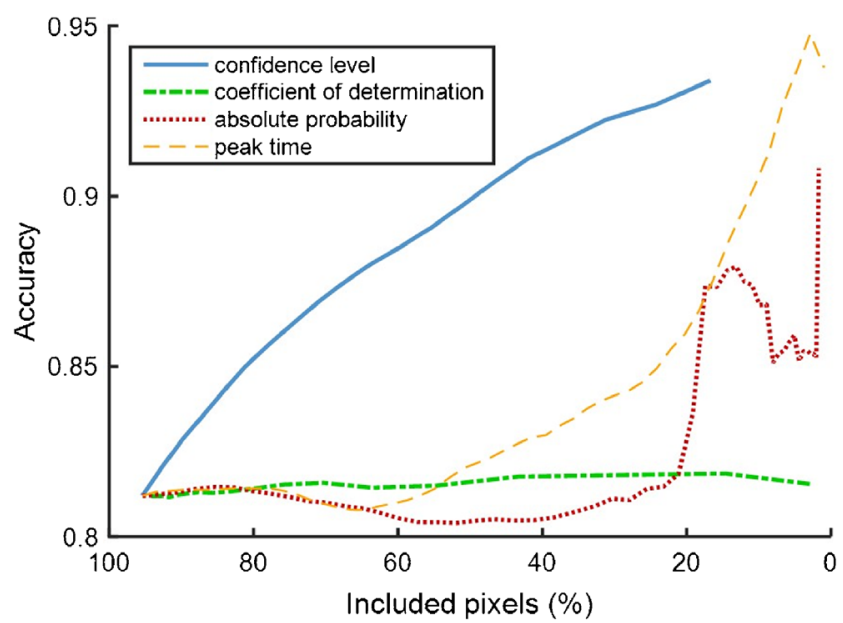

Fig. 1 Accuracy of the Gaussian mixture model classifier by exclusion of pixels based upon their confidence level, coefficient of determination, absolute probability, and peak time. The classifier was run using a set of parameters containing $r, \mu, \kappa$, and PT malignant pixels, as well as over patients and regions; exclusion would, therefore, not result in extra $\mathrm{PCa}$ foci being missed.

To illustrate the results of whole-prostate classification and the effect of pixel exclusion, the US and classification maps as well as histological images of two patients are shown in Figure 3. For the first set in Figure 2, the accuracy and NPV have grown from $81 \pm 11 \%$ to $90 \pm 10 \%$ and from $83 \pm 16 \%$ to $91 \pm 13 \%$ with $51 \pm 17 \%$ of the pixels remaining. These values are $72 \pm 10 \%$ to $90 \pm 7 \%$ and $87 \pm 15 \%$ to $89 \pm 15 \%$ for the second set, again with $51 \pm 14 \%$ of the pixels included. These exclusion percentages include the $4.5 \pm 3.2 \%$ of pixels that could not be fitted by the LDRW model. For reference, Figure 4 depicts the individual, normalized parametric maps that contribute to a multiparametric image.

\section{Discussion}

According to today's guidelines [5], reliable PCa diagnosis requires $\mathrm{a} \geq 10$ core systematic biopsy under US guidance and local anaesthesia. In recent years, an increasing emphasis has been laid on imaging and targeted biopsy [9] in view of the number of reported complications [6], over-diagnoses due to the overestimation of pathologically insignificant lesions [3], and under-diagnoses due to small high-risk PCa foci being missed [8]. Contrast-enhanced US allows the extraction of multiple parameters that have potential to serve as a diagnostic marker for malignancy. The presented multiparametric approach combines perfusion-related parameters from conventional DCE-US and dispersion-related parameters from CUDI by means of a GMM classifier.

The optimal subset of parameters comprises $r, \mu, \kappa$, and PT and thus features parameters from all analysis methods. Of these parameters, $r$ contributes most to the outcome, which is consistent with previous publications on CUDI [39]. As $\mu$ and $\kappa$ jointly describe the shape of LDRW-modelled TIC [55], it is not surprising the combination of these two has the greatest added value to $r$. Finally, the addition of PT offers a slight improvement to the accuracy ( $80 \%$ to $81 \%$ ). Though early enhancement is a strong marker of malignancy, the quantitative use of the PT is normally complicated by its strong dependence on operator and circulation time [34]. In combination with the other parameters, however, the PT is able to further delineate malignant and benign regions. Despite its good performance as a single parameter, the WIT is not included in the multiparametric sets. We expect that this is the result of the high correlation between WIT and $\mu$ (Pearson's $r$ : 0.88), making this parameter redundant after inclusion of $\mu$.

We have shown that observations that are likely to be misclassified can be recognized by their low confidence level. Excluding low-confidence pixels from the multiparametric map increases the reliability of the classification. A confidence 


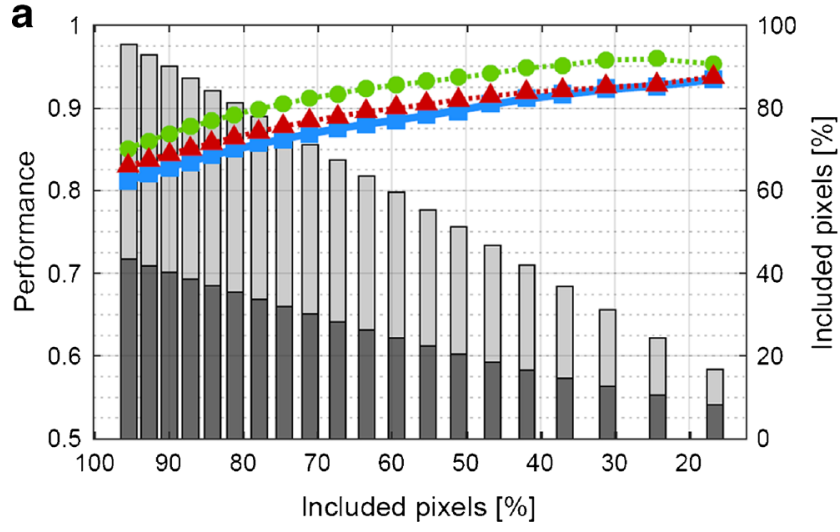

Fig. 2 Performance of the Gaussian mixture model classifier upon exclusion of pixels with the lowest confidence using an increasing confidence threshold for (a) $r, \mu, \kappa$, PT and (b) var, $\mu$, int, $r$. The bars

threshold of 0.5 leads to an average pixel exclusion of 36 $\pm 15 \%$ per prostate (ranging from 8 to $67 \%$ ). In general, the pixel exclusion approach resulted in disregarding pixels in the areas where benign regions border on malignant ones rather

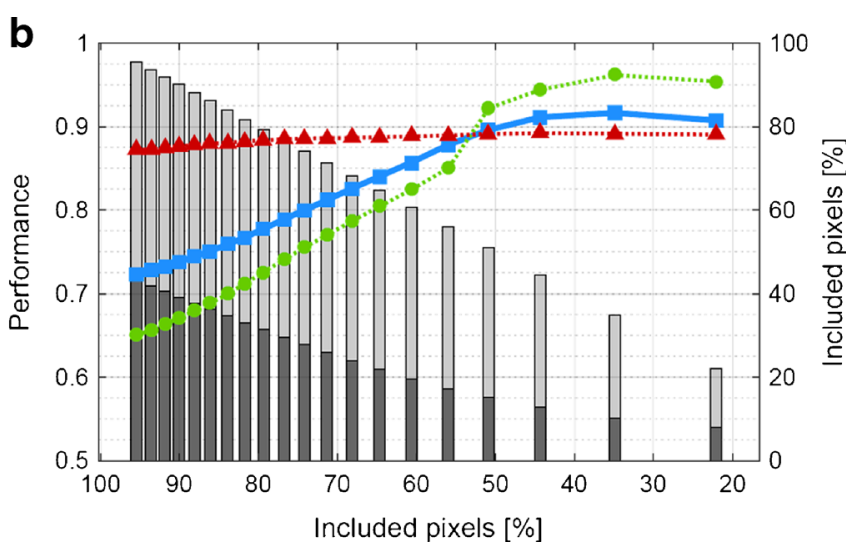

represent the percentage of benign (grey) and malignant (dark) pixels that are still included; the lines represent the evolution of accuracy (blue squares $\boldsymbol{\bullet}$ ), NPV (red triangles $\boldsymbol{\Delta}$ ), and PPV (green circles $\bullet$ )

than pixels in specific prostates. As can be seen in Figure 2, exclusion does not favour malignant or benign pixels specifically. Figure 3 shows typical examples of classification maps. There is a high correspondence between the maps and histology,
Fig. 3 The B-mode transrectal ultrasound, confidence-weighted classification image, exclusionclassification images with a threshold of $P>0.5$ and histopathological images of patient $A(a t / m ~ d)$ and patient $B$, (e $\mathrm{t} / \mathrm{m} \mathrm{h}$ ). In the classification images, red regions are classified as malignant (i.e. suspicious) and green regions as benign (i.e. not suspicious). In the histopathological images, malignant areas are indicated with red. Parameters: $r, \mu, \kappa$, and PT. ROIs are shown in overlay to the B-mode images
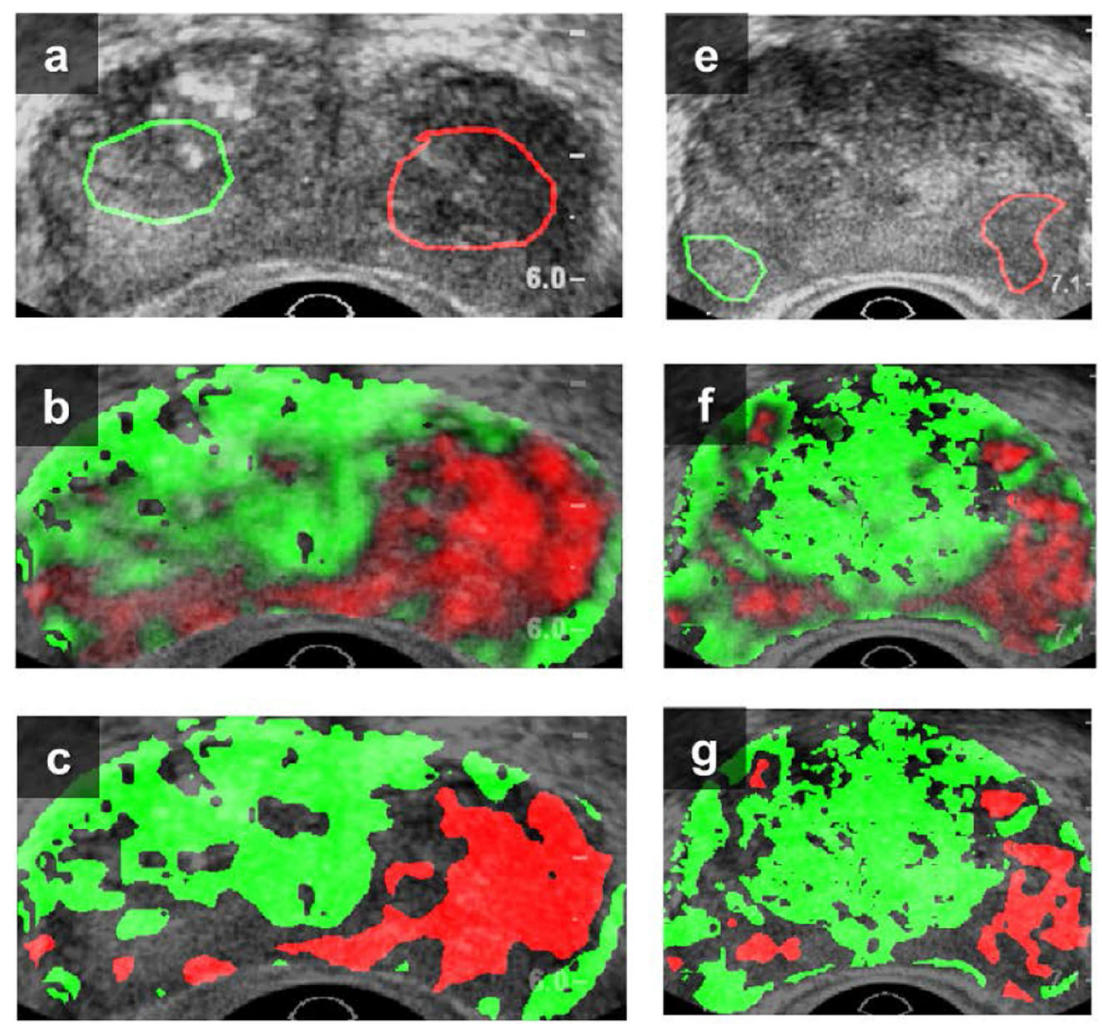

\section{d}

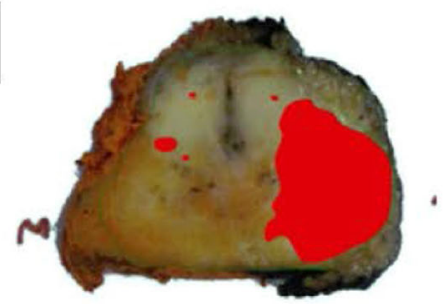

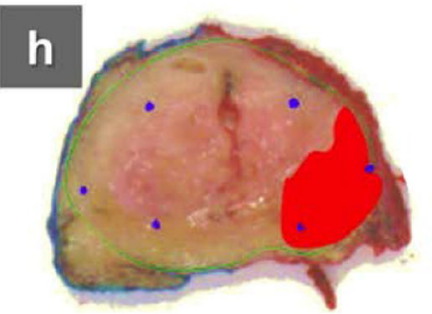


Fig. 4 Example of the four normalized parametric maps that serve as input for the best performing multiparametric map as shown below. All maps overlay the B-mode TRUS image. Red regions are classified as malignant (i.e. suspicious) and green regions as benign (i.e. not suspicious) of which the transparency is scaled with the confidence level. The histology slice with tumour tissue marked red is shown in the upper right corner of the multiparametric image
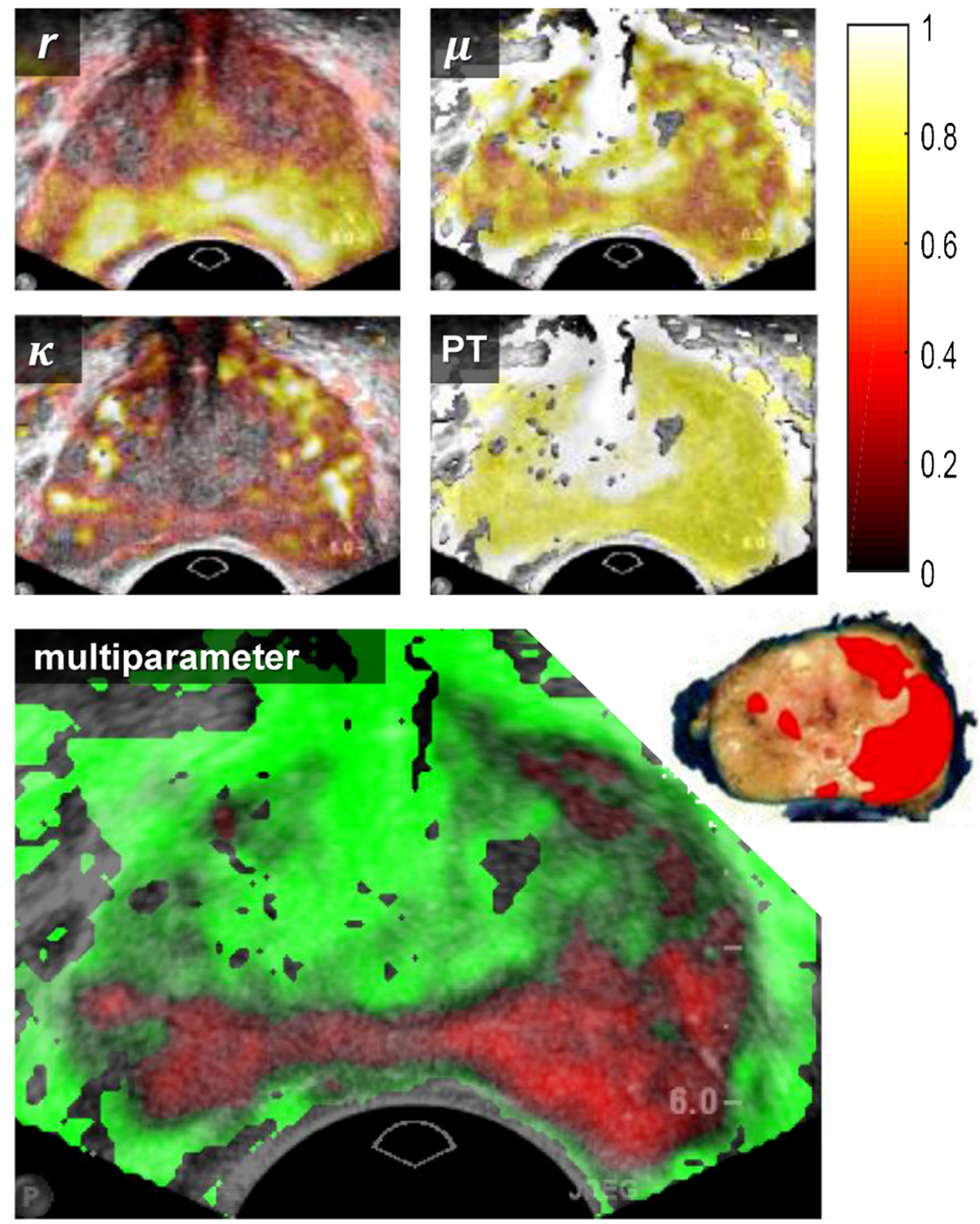

even though small regions remain misclassified after pixel exclusion. Due to the preservation of the correctly classified malignant regions, these results are clinically relevant for, e.g. targeted biopsy. Because the current analysis faces limitations with respect to the registration of US imaging planes with histology slices, we made use of histologically proven ROIs. These ROIs were $\sim 0.5$ $\mathrm{cm}^{2}$ sized, which resembles the critical size of clinically relevant foci [47]. In the future, three-dimensional US models would enable us to apply more accurate registration.

In the current analysis, 9 (20\%) of the 44 regions predominantly ( $>50 \%$ of the pixels) classified as negative were misclassified. In Table 1, Gleason score $3+3$ represents most of these false negative regions. The Gleason score, which comprises the grade of the two most prevalent histological patterns found in a stained prostate tissue slice, is an indicator of the stage and aggressiveness of prostatic carcinoma [56, 57]. Following recent consensus in prostate grading, Gleason rate score $3+3$ is rated as grade group 1 , indicating very low-risk disease with high survival rates and virtually no chance of metastasis $[58,59]$. We believe that the use of a diverse training set (i.e. a set that consists of malignancies with varying aggressiveness) leads to a higher risk of misclassification in prostates containing very low-grade or very high-grade $\mathrm{PCa}$, as these are the most different from the training set average.

The current performance is limited by the small training set in this study, hampering the possibility to define subgroups according to Gleason score. Since the microvascular density is a viable marker in the staging of $\mathrm{PCa}$ [18], this might allow us to distinguish low-risk and high-risk PCa. Studies in contrast-enhanced MRI suggest that perfusion-based discrimination between $\mathrm{PCa}$ grades, and even prostatitis, is possible [60]. In this study, we did not assess the differences in tumour classification of grades; an extended dataset would allow such validation in the future. Another limitation of the study concerns the analysis of small foci, which, considering the error margin in appointing the ROIs, could not be included in the study.

DCE-US is not only a valuable modality for diagnosis of $\mathrm{PCa}$; its use is increasingly mentioned as a tool to monitor the therapeutic effect of focal therapy. For instance, DCE-US was found to map tissue devascularisation as well as DCE-MRI 
after interstitial laser therapy [61, 62]. DCE-US has, therefore, been used for interstitial laser therapy [63] and high-intensity focused US treatment to visualize viable and devascularized regions [64, 65]. Apart from the classifier's aid for tumour localization and monitoring, the ability of such classifier to discriminate low-risk and high-risk disease would especially improve the application of focal therapy and active surveillance strategies.

In conclusion, we see that combined evaluation of contrastenhanced ultrasonographic parameters has superior accuracy and NPV in tumour localization compared to the individual parametric maps. The GMM-based multiparametric analysis is fast, versatile and allows a reliable confidence estimation of its classification. It was shown that pixel exclusion could boost the performance even more without disregarding relevant areas of the prostate. Like in computer-aided diagnosis of breast lesions $[66,67]$, an extensive review of other algorithms is recommended to obtain an overview of the performance, advantages and drawbacks of other classification methods for the detection of PCa. In the future, parametric maps derived from other US modalities such as Doppler and elastography could also be included [68], as well as the results from other diagnostic tools (e.g. PSA assessment), but this is beyond the scope of the current study. Furthermore, this analysis is based on a small patient group, and we recognize that a more extended validation is needed to derive global measures for classification. We expect that this method can also be employed to image other types of cancer.

Acknowledgements The scientific guarantor of this publication is Massimo Mischi. The authors of this manuscript Rogier Wildeboer and Maarten Kuenen declare relationships with the following company: Philips. This study has received funding by the European Research Council Starting Grant (\#280209), an unrestricted grant from the Dutch Cancer Society (\#UVA2013-5941) and the IMPULS2-program within Eindhoven University of Technology in collaboration with Philips. No complex statistical methods were necessary for this paper. Institutional Review Board approval was obtained. Written informed consent was obtained from all patients in this study. Methodology: retrospective, experimental, performed at one institution

Open Access This article is distributed under the terms of the Creative Commons Attribution 4.0 International License (http:// creativecommons.org/licenses/by/4.0/), which permits unrestricted use, distribution, and reproduction in any medium, provided you give appropriate credit to the original author(s) and the source, provide a link to the Creative Commons license, and indicate if changes were made.

\section{References}

1. American Cancer Society (2015) Cancer Facts and Figures 2015. Am Cancer Soc

2. Cooperberg MR, Lubeck DP, Meng MV et al (2004) The changing face of low-risk prostate cancer: trends in clinical presentation and primary management. J Clin Oncol 22:2141-2149

3. Loeb S, Bjurlin MA, Nicholson J et al (2014) Overdiagnosis and overtreatment of prostate cancer. Eur Urol 65:1046-1055
4. Bangma CH, Roemeling S, Schröder FH (2007) Overdiagnosis and overtreatment of early detected prostate cancer. World J Urol 25:3-9

5. Heidenreich A, Bastian PJ, Bellmunt J et al (2014) EAU guidelines on prostate cancer. Part 1: screening, diagnosis, and local treatment with curative intent - update 2013. Eur Urol 65:124-137

6. Loeb S, Vellekoop A, Ahmed HU et al (2013) Systematic review of complications of prostate biopsy. Eur Urol 64:876-892

7. Loeb S, van den Heuvel S, Zhu X et al (2012) Infectious complications and hospital admissions after prostate biopsy in a european randomized trial. Eur Urol 61:1110-1114

8. Ukimura O, Coleman JA, de la Taille A et al (2013) Contemporary role of systematic prostate biopsies: indications, techniques, and implications for patient care. Eur Urol 63:214-230

9. van Hove A, Savoie P-H, Maurin C et al (2014) Comparison of image-guided targeted biopsies versus systematic randomized biopsies in the detection of prostate cancer: a systematic literature review of well-designed studies. World J Urol 32:847-858

10. Heijmink SW, van Moerkerk H, Kiemeney LALM et al (2006) A comparison of the diagnostic performance of systematic versus ultrasound-guided biopsies of prostate cancer. Eur Radiol 16:927-938

11. Ahmed HU, Moore C, Emberton M (2009) Minimally-invasive technologies in uro-oncology: the role of cryotherapy, HIFU and photodynamic therapy in whole gland and focal therapy of localised prostate cancer. Surg Oncol 18:219-232

12. Scheenen TWJ, Rosenkrantz AB, Haider MA, Fütterer JJ (2015) Multiparametric magnetic resonance imaging in prostate cancer management: current status and future perspectives. Invest Radiol 50:594-600

13. de Rooij M, Hamoen EHJ, Fütterer JJ et al (2014) Accuracy of multiparametric MRI for prostate cancer detection: a meta-analysis. Am J Roentgenol 202:343-351

14. Loch AC, Bannowsky A, Baeurle L et al (2007) Technical and anatomical essentials for transrectal ultrasound of the prostate. World J Urol 25:361-366

15. Smeenge M, de la Rosette JJMCH, Wijkstra H (2012) Current status of transrectal ultrasound techniques in prostate cancer. Curr Opin Urol 22:297-302

16. Russo G, Mischi M, Scheepens W et al (2012) Angiogenesis in prostate cancer: onset, progression and imaging. BJU Int 110:794-808

17. Carmeliet P, Jain RK (2000) Angiogenesis in cancer and other diseases. Nature 407:249-257

18. Brawer MK (1996) Quantitative microvessel density: a staging and prognostic marker for human prostatic carcinoma. Cancer 78:345-349

19. Halpern EJ, Strup SE (2000) Using gray-scale and color and power doppler sonography to detect prostatic cancer. Am J Roentgenol 174:623-627

20. Mitterberger MJ, Aigner F, Horninger W et al (2010) Comparative efficiency of contrast-enhanced colour Doppler ultrasound targeted versus systematic biopsy for prostate cancer detection. Eur Radiol 20:2791-2796

21. Cosgrove D (2003) Angiogenesis imaging-ultrasound. Br J Radiol 60:324-330

22. Halpern EJ, McCue PA, Aksnes AK et al (2002) Contrast-enhanced US of the prostate with sonazoid: comparison with whole-mount prostatectomy specimens in 12 patients. Radiology 222:361-366

23. Matsumoto K, Nakagawa K, Hashiguchi A et al (2010) Contrastenhanced ultrasonography of the prostate with Sonazoid. Jpn J Clin Oncol 40:1099-1104

24. Li Y, Tang J, Fei X, Gao Y (2013) Diagnostic performance of contrast enhanced ultrasound in patients with prostate cancer: a meta-analysis. Acad Radiol 20:156-164

25. Delorme S, Knopp MV (1998) Non-invasive vascular imaging: assessing tumour vascularity. Eur Radiol 8:517-527

26. Trabulsi EJ, Sackett D, Gomella LG, Halpern EJ (2010) Enhanced transrectal ultrasound modalities in the diagnosis of prostate cancer. Urology 76:1025-1033 
27. Wink M, Frauscher F, Cosgrove D et al (2008) Contrast-enhanced ultrasound and prostate cancer; a multicentre european research coordination project. Eur Urol 54:982-993

28. Halpern EJ, Verkh L, Forsberg F et al (2000) Initial experience with contrast-enhanced sonography of the prostate. Am J Roentgenol 174:1575-1580

29. Seitz M, Gratzke C, Schlenker B et al (2011) Contrast-enhanced transrectal ultrasound (CE-TRUS) with cadence-contrast pulse sequence (CPS) technology for the identification of prostate cancer. Urol Oncol Semin Orig Investig 29:295-301

30. Qi T, Chen Y, Zhu Y et al (2014) Contrast-enhanced transrectal ultrasonography for detection and localization of prostate index tumor: correlation with radical prostatectomy findings. Urology 84:138-143

31. Postema AW, Frinking PJA, Smeenge M et al (2015) Dynamic contrast-enhanced ultrasound parametric imaging for the detection of prostate cancer. BJU Int 117:598-603

32. Elie N, Kaliski A, Péronneau P et al (2007) Methodology for quantifying interactions between perfusion evaluated by DCE-US and hypoxia throughout tumor growth. Ultrasound Med Biol 33:549-560

33. Eckersley RJ, Sedelaar JP, Blomley MJK et al (2002) Quantitative microbubble enhanced transrectal ultrasound as a tool for monitoring hormonal treatment of prostate carcinoma. Prostate 51:256-267

34. Tang J, Yang J-C, Luo Y et al (2008) Enhancement characteristics of benign and malignant focal peripheral nodules in the peripheral zone of the prostate gland studied using contrast-enhanced transrectal ultrasound. Clin Radiol 63:1086-1091

35. Qi TY, Chen YQ, Jiang J et al (2013) Contrast-enhanced transrectal ultrasonography: Measurement of prostate cancer tumor size and correlation with radical prostatectomy specimens. Int J Urol 20:1085-1091

36. Eckersley RJ, Cosgrove DO, Blomley MJ, Hashimoto H (1998) Functional imaging of tissue response to bolus injection of ultrasound contrast agent. In: Ultrason. Symp. 1998. Proceedings., 1998 IEEE. IEEE, pp 1779-1782

37. Kuenen MPJ, Mischi M, Wijkstra H (2011) Contrast-ultrasound diffusion imaging for localization of prostate cancer. IEEE Trans Med Imaging 30:1493-1502

38. Mischi M, Kuenen MPJ, Wijkstra H (2012) Angiogenesis imaging by spatiotemporal analysis of ultrasound contrast agent dispersion kinetics. IEEE Trans Ultrason Ferroelectr Freq Control 59:621-629

39. Kuenen MPJ, Saidov TA, Wijkstra H et al (2013) Spatiotemporal correlation of ultrasound contrast agent dilution curves for angiogenesis localization by dispersion imaging. IEEE Trans Ultrason Ferroelectr Freq Control 60:2665-2669

40. Clements R (2002) The role of transrectal ultrasound in diagnosing prostate cancer. Curr Urol Rep 3:194-200

41. Fawcett T (2006) An introduction to ROC analysis. Pattern Recognit Lett 27:861-874

42. Metz CE (1978) Basic principles of ROC analysis. Semin Nucl Med 8:283-298

43. Chang R-F, Wu W-J, Moon WK et al (2003) Support vector machines for diagnosis of breast tumors on US images. Acad Radiol 10:189-197

44. Loch T, Leuschner I, Genberg C et al (1999) Artificial neural network analysis (ANNA) of prostatic transrectal ultrasound. Prostate 39:198-204

45. Acharya R, Ng EYK, Chang YH et al (2008) Computer-based identification of breast cancer using digitized mammograms. J Med Syst 32:499-507

46. Greis C (2004) Technology overview: SonoVue. Eur Radiol Suppl 14:P11-P15

47. Montironi R, van der Kwast T, Boccon-Gibod L et al (2003) Handling and pathology reporting of radical prostatectomy specimens. Eur Urol 44:626-636
48. Schalk SG, Demi L, Smeenge M et al (2015) 4-D Spatiotemporal analysis of ultrasound contrast agent dispersion for prostate cancer localization : a feasibility study. IEEE Trans Ultrason Ferroelectr Freq Control 62:839-851

49. Mischi M, Kalker A, Korsten HHM (2004) Moment method for the local density random walk model interpolation of ultrasound contrast agent dilution curves. In: Proc. 17th Int. EURASIP Conf. BIOSIGNAL 2004, Brno (Czech Rep.), Jun. 23-25. Citeseer, pp 33-35

50. Fraley C, Raftery AE (2002) Model-based clustering, discriminant analysis, and density estimation. J Am Stat Assoc 97:611-631

51. Banfield JD, Raftery AE (1993) Model-based Gaussian and nonGaussian clustering. Biometrics 49:803-821

52. Han SH, Ackerstaff E, Stoyanova R et al (2013) Gaussian mixture model-based classification of dynamic contrast enhanced MRI data for identifying diverse tumor microenvironments: preliminary results. NMR Biomed 26:519-532

53. Grim J, Somol P, Haindl M, Daneš J (2009) Computer-aided evaluation of screening mammograms based on local texture models. Image Process IEEE Trans 18:765-773

54. Redner R, Walker H (1984) Mixture densities, maximum likelihood and the EM algorithm. SIAM Rev 26:195-239

55. Strouthos C, Lampaskis M, Sboros V et al (2010) Indicator dilution models for the quantification of microvascular blood flow with bolus administration of ultrasound contrast agents. Ultrason Ferroelectr Freq Control IEEE Trans 57:1296-1310

56. Gleason DF (1992) Histologic grading of prostate cancer: a perspective. Hum Pathol 23:273-279

57. Humphrey PA (2004) Gleason grading and prognostic factors in carcinoma of the prostate. Mod Pathol 17:292-306

58. Kryvenko ON, Epstein JI (2015) Changes in prostate cancer grading: Including a new patient-centric grading system. Prostate 76:427-433

59. Epstein J, Egevad L, Amin MB et al (2016) The 2014 International Society of Urological Pathology (ISUP) consensus conference on Gleason grading of prostatic carcinoma: definition of grading patterns and proposal for a new grading system. Am J Surg Pathol 40:244-252

60. Franiel T, Lüdemann L, Rudolph B et al (2008) Evaluation of normal prostate tissue, chronic prostatitis, and prostate cancer by quantitative perfusion analysis using a dynamic contrast-enhanced inversion-prepared dual-contrast gradient echo sequence. Invest Radiol 43:481-487

61. Atri M, Gertner MR, Haider MA et al (2009) Contrast-enhanced ultrasonography for real-time monitoring of interstitial laser thermal therapy in the focal treatment of prostate cancer. Can Urol Assoc J 3:125-130

62. van den Bos W, Bruin DM, Randen A et al (2016) MRI and contrastenhanced ultrasound imaging for evaluation of focal irreversible electroporation treatment: results from a phase I-II study in patients undergoing IRE followed by radical prostatectomy. Eur Radiol 26:22522560

63. Colin P, Mordon S, Nevoux P et al (2012) Focal laser ablation of prostate cancer: definition, needs, and future. Adv Urol 2012:589160

64. Rouvière O, Glas L, Girouin N et al (2011) Prostate cancer ablation with transrectal high-intensity focused ultrasound: assessment of tissue destruction with contrast-enhanced US. Radiology 259:583-591

65. Crouzet S, Rouviere O, Martin X, Gelet A (2014) High-intensity focused ultrasound as focal therapy of prostate cancer. Curr Opin Urol 24:225-230

66. Nattkemper TW, Arnrich B, Lichte O et al (2005) Evaluation of radiological features for breast tumour classification in clinical screening with machine learning methods. Artif Intell Med 34:129-139

67. Jalalian A, Mashohor SBT, Mahmud HR et al (2013) Computeraided detection/diagnosis of breast cancer in mammography and ultrasound: a review. Clin Imaging 37:420-426

68. Postema A, Mischi M, de la Rosette J, Wijkstra H (2015) Multiparametric ultrasound in the detection of prostate cancer: a systematic review. World J Urol 33:1651-1659 